\title{
Lisdexamfetamine Dimesylate
}

National Cancer Institute

\section{Source}

National Cancer Institute. Lisdexamfetamine Dimesylate. NCI Thesaurus. Code C76628.

The dimesylate form and prodrug of the d-isomer of amphetamine, a non-

catecholamine sympathomimetic amine with central nervous system (CNS) stimulating activity. Upon administration, lisdexamphetamine is converted to dextroamphetamine through cleavage of the lysine group. Dextroamphetamine acts by facilitating the release of catecholamines, particularly noradrenaline and dopamine, from its storage sites in nerve terminals in the CNS, and inhibits their uptake within the mesocorticolimbic system, a major component of the brain reward system, resulting in measurable behavioral changes such as euphoria, mental alertness and excitement and appetite suppression. As a CNS stimulant, this agent may increase blood pressure. 